\title{
Parametric relaxation in whispering gallery mode exciton-polariton condensates
}

\author{
C. P. Dietrich, ${ }^{1,2,{ }^{*}}$ R. Johne, ${ }^{3}$ T. Michalsky, ${ }^{1}$ C. Sturm,${ }^{1}$ P. Eastham, ${ }^{4}$ H. Franke,${ }^{1}$ M. Lange, ${ }^{1}$ \\ M. Grundmann, ${ }^{1}$ and R. Schmidt-Grund ${ }^{1, \dagger}$ \\ ${ }^{1}$ Universität Leipzig, Institut für Experimentelle Physik II, Linnéstrasse 5, 04103 Leipzig, Germany \\ ${ }^{2}$ SUPA, School of Physics and Astronomy, University of St. Andrews, KY16 9SS, St. Andrews, Scotland, United Kingdom \\ ${ }^{3}$ Max Planck Institute for the Physics of Complex Systems, Nöthnitzer Strasse 38, 01187 Dresden, Germany \\ ${ }^{4}$ Trinity College Dublin, College Green, Dublin 2, Ireland \\ (Received 21 July 2014; revised manuscript received 11 November 2014; published 16 January 2015)
}

\begin{abstract}
Polariton condensation of one-dimensional multimode whispering gallery mode exciton polaritons is investigated in hexagonal $\mathrm{ZnO}$ microwires at cryogenic temperatures. At threshold, stimulated scattering is fed by the resonant emission from highly populated defect-bound excitons. With further increasing excitation power, condensates relax within the multimode whispering gallery mode polariton ladder, leading to their effective evaporative cooling. The relaxation is a parametric polariton-polariton scattering process evidenced by its intensity, energy, and momentum evolution. The experimental observations are in good agreement with numerical simulations based on a model for polariton-polariton scattering extended to the multimodal whispering gallery mode system.
\end{abstract}

DOI: 10.1103/PhysRevB.91.041202

The strong light-matter coupling regime of spatially confined photons in a Fabry-Pérot microcavity coupled to a reservoir of electronic states results in the formation of new eigenstates, so-called cavity exciton polaritons. Their dispersion close to zero wave vector provides an energetic trap into which polaritons can relax efficiently. A massive occupation of those states would lead to Bose-Einstein condensation (BEC) that has intensively been studied in recent years, thereby showing remarkable properties such as superfluidity [1], integer [2] and half-integer quantized vortices [3], or Bogoliubov excitations [4]. Multimode polariton systems that undergo inter- or intrabranch parametric scattering processes [5-7] are promising for sources of entangled photons [8-10]. However, using those effects in integrated optical circuits requires manipulability and guidability that is more easily achieved in one-dimensional microcavities. A major step towards this goal has recently been demonstrated in polariton microcavities etched into wire-shaped structures resulting in the formation of one-dimensional polariton condensates with long polariton lifetimes and long propagation lengths [11]. Although parametric oscillation processes have already been demonstrated in those structures [6], a richer mode structure is necessary for the creation of entangled polaritons [8]. Our "bottom up" approach for the creation of one-dimensional multimode polariton systems combines the excellent photonic properties of whispering gallery modes (WGMs) with the outstanding electronic properties of excitons in hexagonal $\mathrm{ZnO}$ microwires. Beneficially, $\mathrm{ZnO}$ microwires can easily be grown by carbothermal vapor-phase transport [12] and naturally provide strong photonic confinement by total internal reflection (TIR) [13]. Hence, they obviate the need for highly reflecting Bragg mirrors and sophisticated fabrication steps. One-dimensional WGM exciton-polariton condensates in those $\mathrm{ZnO}$ microwires then benefit from the multimodal character of the photonic component along with a long photon lifetime.

\footnotetext{
*cpd3@st-andrews.ac.uk

${ }^{\dagger}$ schmidt-grund@physik.uni-leipzig.de
}

\section{PACS number(s): 71.36.+c, 42.55.Sa, 78.55.Et}

Here, we demonstrate the condensation of WGM exciton polaritons at cryogenic temperature being accompanied by complex parametric polariton scattering processes. We observe an efficient exciton relaxation into defect-bound states, whose radiative decay resonantly excites the polariton condensate above threshold in one of the many lower polariton branches. By increasing the excitation power, condensate relaxation into further lower polariton states takes place, providing an efficient evaporative cooling mechanism. We show experimentally and theoretically that this process is caused by multichannel scattering mechanisms leading to parametric mixing of polariton states at higher excitation densities, as evidenced by constant energy and momentum steps between the different polariton states. We believe that these multimode parametric mixing processes pave the way towards polariton entanglement.

Figure 1 shows typical angle-resolved photoluminescence (PL) images (cw excitation) of a $\mathrm{ZnO}$ microwire recorded at $10 \mathrm{~K}$. Besides prominent transitions of free excitons $\left(\mathrm{X}^{\mathrm{A}}\right)$, donor-bound excitons $\left(\mathrm{D}^{0}, \mathrm{X}\right)$ [14], and defect-bound excitons (DBX) [15], it clearly shows dispersion behavior of confined modes evidenced by the increase of the mode energy with the emission angle, or equivalently the wave vector. Note that the PL image is recorded unpolarized and shows contributions from transverse electric (TE)- and transverse magnetic (TM)polarized modes. In order to assess if WGMs and $\mathrm{ZnO}$ excitons strongly interact with each other, a careful remodeling of the photonic WGM dispersion curves is indispensable. In our case, we determine an average coupling strength of around $100 \mathrm{meV}$ between $\mathrm{ZnO}$ excitons and WGM photons (see Ref. [16] for details), which is in very good agreement with earlier publications [17]. Figure 1 shows that the WGM exciton-polariton system is multimodal, i.e., several photonic WGMs with different axial mode numbers strongly couple to the excitonic transitions [18]. In the present case, the WGM ground state $\left(N_{\mathrm{WGM}}=1\right)$ has an energy far below the exciton resonance (which is close to $N_{\mathrm{WGM}} \approx 200$ ), thus it does not play any role in the strong interaction between cavity photons and excitons.

Excitation-dependent PL images (pulsed excitation) recorded at $T=10 \mathrm{~K}$ from a $\mathrm{ZnO}$ microwire are shown 
(a)

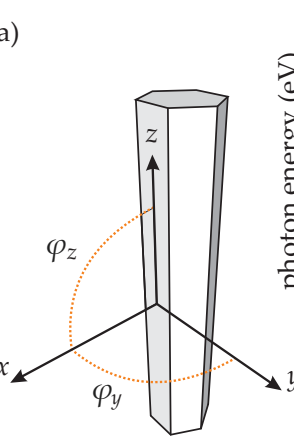

\section{(b)}

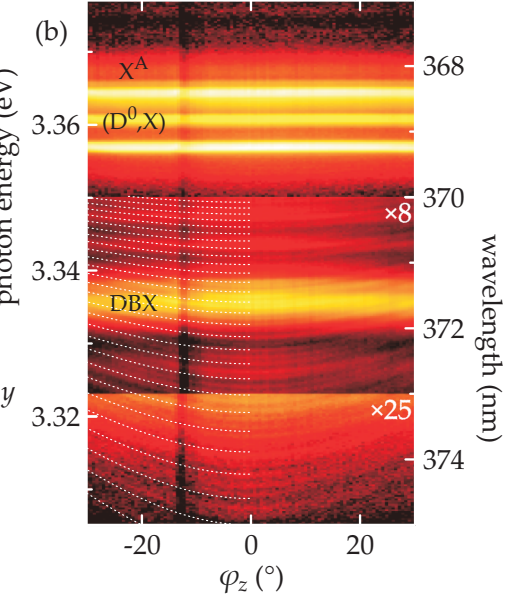

FIG. 1. (Color online) (a) Microwire coordinate system: The $x y$ plane coincides with the cross section plane, and the wire axis coincides with the $z$ axis. Angles are defined accordingly. (b) PL image of a $\mathrm{ZnO}$ microwire recorded at cryogenic temperature. Respective ZnO-related transitions are labeled: free $(\mathrm{X})$, donor-bound $\left(\mathrm{D}^{0}, \mathrm{X}\right)$, and defect-bound (DBX) excitons. The white dotted lines indicate modeled dispersion curves for TE-polarized polaritons.

in Fig. 2 (top). Below threshold, transitions of donor-bound excitons dominate the PL and a weak emission is visible around $3.337 \mathrm{eV}$, which can be related to defect-bound excitons (DBX). When increasing the excitation intensity to $P_{0}=$ $15 \mathrm{~kW} / \mathrm{cm}^{2}$, the system reaches the lasing regime (evidenced by the buildup of spatial coherence-see Ref. [16]). At threshold, emission around the DBX transition becomes more pronounced and even dominating at further high excitation power. Simultaneously, more peaks appear at energies above and below this initial state as well as at the spectral positions of the polariton states. A closer look at these peaks (see the white-framed inset in Fig. 2, top) reveals that the emission clearly arises from two spots symmetrically shifted from $k=0$ with the following two properties: The emission does not originate from the dispersion minimum but is blueshifted by $\Delta E_{B}=0.3 \mathrm{meV}$ and arises at a finite and discrete wave vector $k_{\|} \approx \pm 0.01 \mu \mathrm{m}^{-1}$. Both properties are strong indications for polariton condensation and can be clearly distinguished from the photon lasing regime (see Ref. [16] for details). The finite $k_{\|}$indicates finite momentum of the involved quasiparticles, gained either by scattering processes or by acceleration along the wire axis away from an inhomogeneous potential landscape $[11,19]$. The latter can be ruled out since our excitation spot size is much larger than the field of view of our microscope setup (please see Ref. [16] for setup details). We therefore do not expect an inhomogeneous background potential induced by the photogenerated exciton reservoir to be present in the collection area.

It can be seen in Fig. 2 that almost no WGM polariton states are occupied at energies above the initial condensate state (around $3.337 \mathrm{eV}$ ). Apart from that, this energy only matches the emission from DBX and does not coincide with any free-exciton- or phonon-assisted process, which is why we exclude any relaxation process involving excitondonor bound exciton, exciton-phonon, polariton-phonon, or interbranch polariton-polariton scattering from the highest lower polariton branch (respectively the free exciton reservoir) into this state. Instead, we propose that the initial condensate state is resonantly excited by the radiative decay of DBX, which are highly occupied at low temperatures due to efficient and fast energy relaxation of free excitons [20,21]. This is a very efficient process since DBX within the whole excitation volume contribute to the excitation of the surface near WGM polariton states.

By further increasing the excitation power, polariton states at lower energies also turn into the condensation regime, i.e., the polariton condensate relaxes into energetically lower polariton states (as can also be seen in momentum space [16]). A remarkable feature of the relaxation process is the increase in the condensate wave vector. That this relaxation can take place at all is a consequence of the multimodality of the WGM exciton-polariton system [13]. In contrast to common semiconductor microcavities where polaritons relax into the system's ground state and experience a strong interparticle interaction, polaritons in multimode systems efficiently relax into lower states when occupation of one of the initial state is reached [22]. Thus, the occupation of each of the initial states is almost clamped to their particular threshold values. As a consequence, the polariton-polariton interaction-driven blueshift of the condensate energy in each of the discrete condensate states is much smaller compared to single mode systems and is almost independent of the excitation power. In the experiment, $\Delta E_{\mathrm{B}}$ is found to be nearly constant above threshold, which reflects this behavior, but also means that the total charge carrier density does not increase further and a quasisteady state is reached. In principle, the energetically lowest target state which can be reached by such a relaxation process should be set by the coupling strength between the exciton and WGM photon as well as their lifetimes.

We simplify the complex process by a scheme depicted in Fig. 2 (bottom) showing a magnified view of the condensation region. Due to an increase of occupation of the initially fed states (around the DBX energy), polariton scattering into the surrounding states sets in. However, condensation is reached first in the initial state at $k_{\|} \approx 0$. With further increasing density, the repulsive interaction between polaritons becomes efficient and polariton-polariton scattering into several branches is observed. Hereby, two polaritons scatter out of the initial state into states with higher as well as lower energy and higher momentum. Since polaritons with higher energy are more excitonlike than lower-energy polaritons and therefore possess a shorter lifetime (in the presented microwire the excitonic lifetime at $4 \mathrm{~K}$ is $\approx 500$ fs compared to a 1-2 ps photonic lifetime), high-energy particles are effectively removed from the system. This process can be understood as an evaporative cooling of the condensate. Besides that, fast relaxation from high-energy states into the initial states or low-energy states can take place. This is supported by the strong suppression of emission above the DBX energy.

The process of relaxation is further analyzed in a magnified section of the PL image with highest excitation of Fig. 2 (top) and shown in Fig. 3(a) together with the calculated TE-polarized polariton dispersion curves (white dotted lines). It is obvious that only TE-polarized polaritons undergo condensation and relaxation whereas TM-polarized modes 


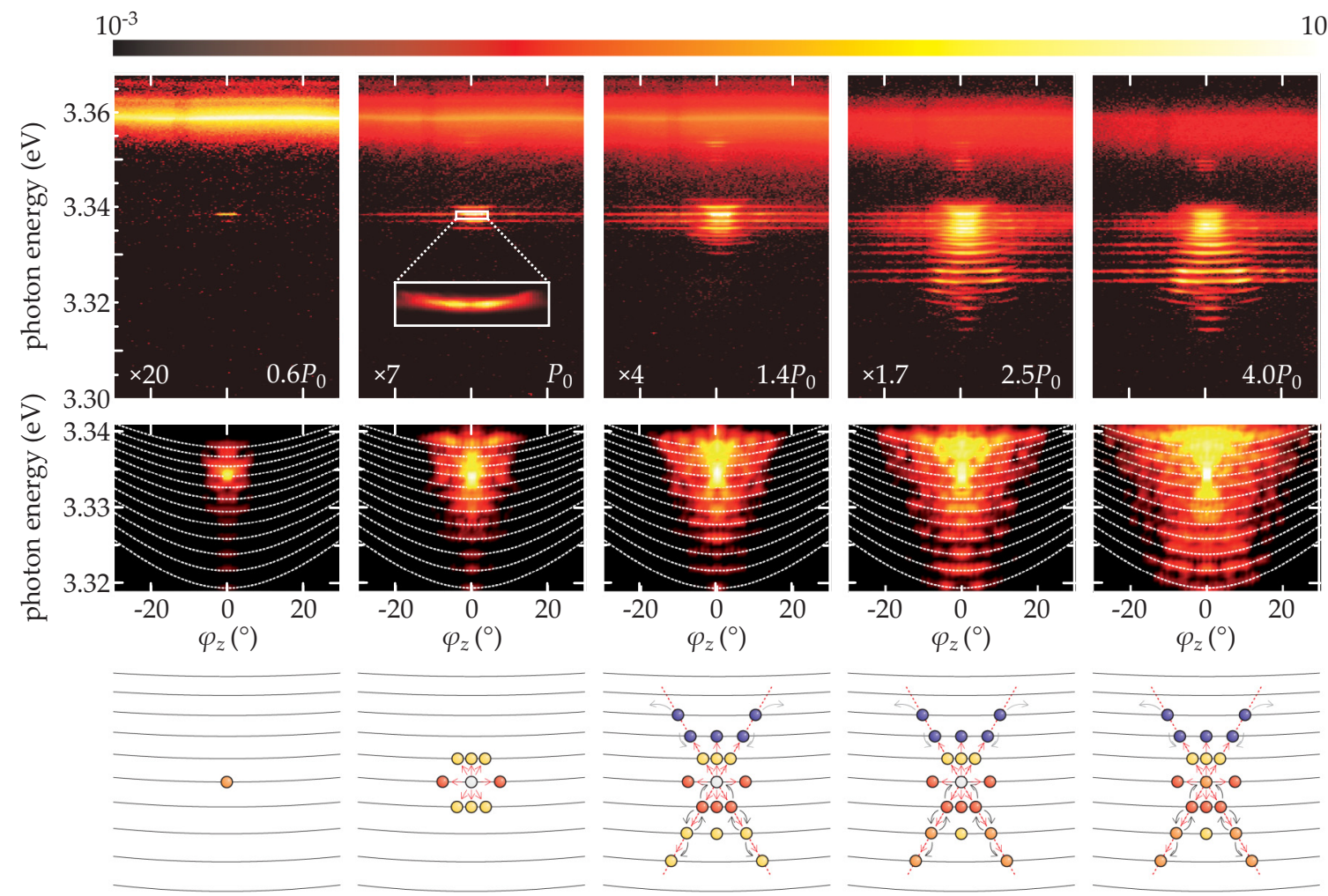

FIG. 2. (Color online) Top: Excitation-dependent, normalized PL images $(T=10 \mathrm{~K})$ of a $\mathrm{ZnO}$ microwire $\left(P_{0}=15 \mathrm{~kW} / \mathrm{cm}^{2}\right)$. Relative excitation powers as well as intensity multiplication factors are given at the bottom of each image. The white frame shows a magnified image of the condensation region at threshold. Middle: Simulated polariton relaxation for increasing pump powers $F_{p}=\{0.1,0.2,0.3,0.4,0.5\}$ in arbitrary units (left to right). The WGM polariton dispersions are shown by the dashed lines. Bottom: Schematic of the excitation power-dependent scattering processes. Note that only one out of many scattering paths is shown for clarity. Occupied polariton states are shown as circles and the color code from light orange to red indicates increasing occupation density. The blue circles indicate target states not visible in the PL images due to their fast decay and relaxation. Red arrows refer to linear polariton-polariton scattering while black arrows indicate parametric mixing processes.

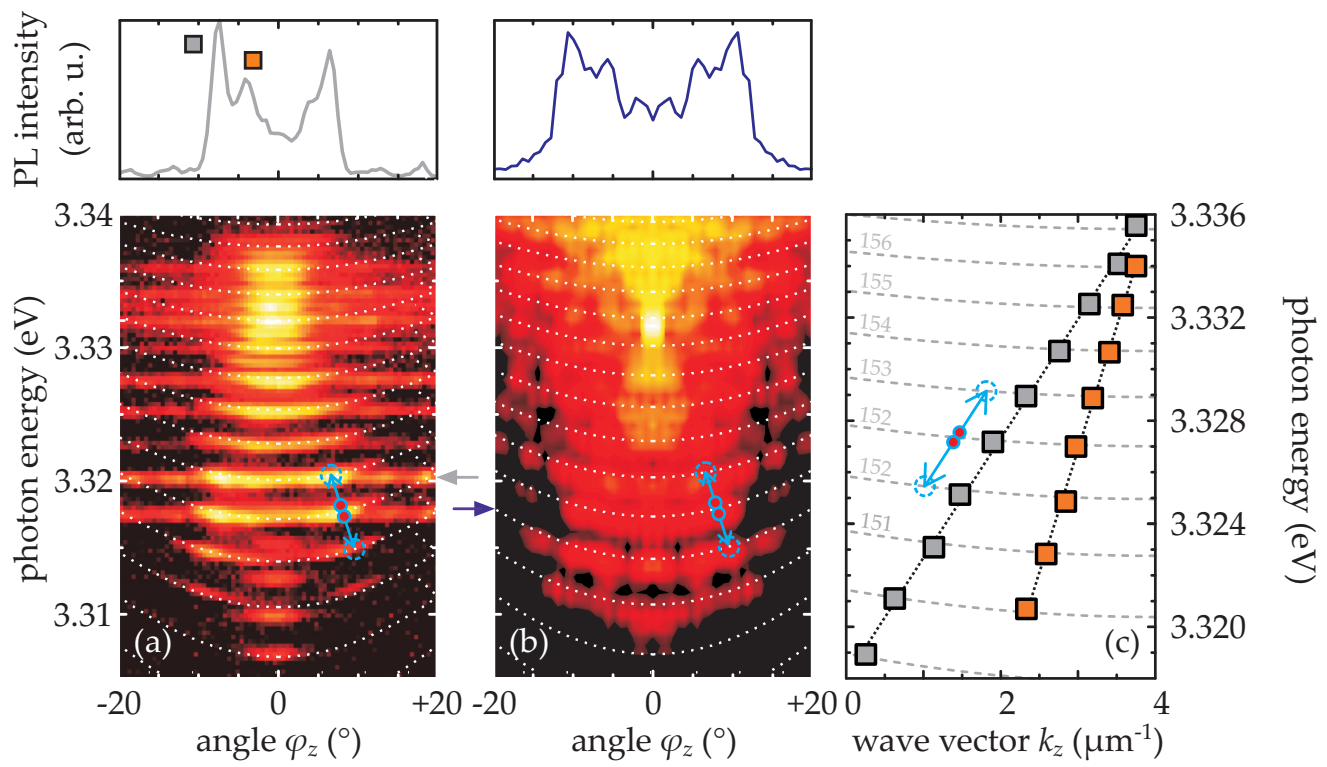

FIG. 3. (Color online) (a) PL image and (b) simulation from Fig. 2 for highest excitation $\left(P=2.8 P_{0}\right)$ in a magnified view. The dotted white lines represent the calculated, TE-polarized WGM exciton-polariton dispersion curves. Above: Momentum profile of image below. The profile is shown for the spectral position marked by the gray and purple arrow. Note that the asymmetry in the emission pattern for positive and negative emission angles might be caused by the slight posture of the wire. (c) Extracted energies and momenta of the experimental polariton condensate states. The linear, dotted black lines are guide to the eyes. The gray dotted lines are calculated dispersion curves. The respective polariton mode numbers are given on the left side. Possible parametric scattering events are sketched by blue arrows in (a)-(c). 
show some emission at the bottom of the dispersion near zero wave vector. This is reasonable since two-dimensional photons (propagating in the cross section plane) only couple to three-dimensional excitons with a suitable wave vector and polarization. Since highly occupied A-exciton states in $\mathrm{ZnO}$ are mainly TE polarized [23], they predominantly couple to TE-polarized photons to form polaritons. Our observation is also consistent with Ref. [24], where only TE-polarized WGMs switch to the photonic lasing regime.

Having a closer look at a momentum profile [see Fig. 3(a), top] integrated over a narrow energy range around one of the condensate levels (at $\approx 3.3262 \mathrm{eV}$ ), we see that at least two pronounced relaxation paths exist (highlighted by squares). For analysis, we extracted the energy and momentum values of all these condensate states and plotted them in Fig. 3(c). It can be seen that both relaxation paths are linear below a certain energy, revealing constant energy and momentum steps between the individual polariton states. The observed constant energy and momentum steps between adjacent states indicate parametric polariton-polariton scattering to be dominant above threshold. This process sets in when the population of distinct initial and target states is high enough. The energy-momentum conservation condition thereby is fulfilled at least for two relaxation paths, with similar merging points of the condensate ladders slightly below the initial state. The complex scattering behavior is also reflected in the observed complex, noncontinuous intensity evolution of the individual states (not shown). Some possible scattering events of the two processes are sketched in Fig. 2 (bottom).

In order to theoretically substantiate our experimental observations, we deduced a model that is able to describe polariton-polariton scattering events in a multimodal polariton system. The model is based on equations derived in Refs. $[25,26]$ and is extended to the whispering gallery mode system with its multimodal structure. The Hamiltonian in the polariton basis reads

$$
H=H_{L P}+H_{P P}+H_{F} .
$$

The first term $H_{L P}$ is the free evolution of the polaritons,

$$
H_{L P}=\sum_{i=1}^{N} \sum_{k} E_{L P}^{(i)}(k) p_{k, i}^{+} p_{k, i},
$$

where $N$ is the number of lower polariton branches in the WGM resonator and $p_{k, i}^{+}\left(p_{k, i}\right)$ is the polariton creation (annihilation) operator for the polaritons with wave vector $k$ in the polariton branch $i$. The polariton-polariton interaction is described by

$$
H_{P P}=\sum_{h, i, j}^{N} \sum_{k, k^{\prime}, q} V_{k, k^{\prime}, q}^{h i j} p_{k+q, i}^{+} p_{k^{\prime}-q, j}^{+} p_{k, h} p_{k^{\prime}, h} .
$$

The interaction potential $V$ comes from the exciton-exciton interaction and can be written as

$$
V_{k, k^{\prime}, q}^{h i j}=\frac{\lambda_{X}}{A} \frac{6 e^{2}}{\varepsilon} X_{k+q, i} X_{k^{\prime}-q, j} X_{k, h} X_{k^{\prime}, h},
$$

where $X$ is the excitonic fraction of the corresponding polariton state and $\lambda_{X}$ is the exciton Bohr radius $(2.87 \mathrm{~nm})$. The excitation area is denoted by $A$. Note that we neglect the saturation of the exciton and consider solely a linear coupling, in contrast to Refs. [25,26]. Finally, we consider the polariton-driving field interaction, which is given by

$$
H_{F}=\sum_{k, i} \Omega_{p r}(t) C_{k, i} p_{k, i}^{+}+\Omega_{p}(t) C_{k_{0}, i_{0}} p_{k_{0}, i_{0}}^{+}+\text {H.c. }
$$

The first term corresponds to a weak probe field $\Omega_{p r}$ incident on all polariton states, which represents a small thermal population. This is required to initiate the parametric scattering process. The second term is a strong pump field $\Omega_{p}$ acting only on a single polariton state with $k_{0}$ and in the branch $i_{0}$, which represents the resonant feeding of the polariton mode by the defect-bound exciton. The terms are weighted by the cavity fraction $C$ of the corresponding state.

The optical response of the probe beam is given by the expectation value $P_{k-q, j}=\left\langle p_{k-q, j}\right\rangle$ which couples through polariton-polariton interactions to the state $P_{k, h}=\left\langle p_{k, h}\right\rangle$. The polariton-polariton diffusion produces a wave-mixing component in one of the polariton branches at wave vector $k+q$. Of course this only holds if the energy $\left[E_{L P}^{(i)}(k+q)+\right.$ $\left.E_{L P}^{(j)}(k-q)-2 E_{L P}^{(h)}(k)=0\right]$ is conserved. Note that $\left|P_{k, i}\right|^{2}$ represents the polariton occupation with wave vector $k$ in mode $i$. While in the typical case with a single lower polariton branch only one configuration of $k$ and $q$ fulfills the above requirement of energy and momentum conservation, a multimodal structure leads to a variety of possible scattering channels. In addition to momentum and energy conservation, parity conservation also needs to be taken into account in multimodal systems [5].

The system reaches even more complexity as each of the polariton modes, populated by parametric scattering, can be a starting point for a new parametric process. The full system of equations for the polariton state amplitudes by factorizing all many-operator expectation values of $\left\langle p_{k, h}\right\rangle,\left\langle p_{k-q, j}\right\rangle$, and $\left\langle p_{k+q, i}\right\rangle$ can finally be written as

$$
\begin{aligned}
\frac{d P_{k, i}}{d t}= & \frac{i}{\hbar}\left(\left[E_{L P}^{(i)}(k)+i \gamma_{k, i}\right] P_{k, i}+F_{p r}+\delta_{k, k_{0}} F_{p}\right. \\
& +\sum_{q, j, h} 2 E_{i} P_{k, i}^{*} P_{k+q, j} P_{k-q, h} \\
& \left.+\sum_{q^{\prime}, j, h} E_{i} P_{k+q^{\prime}, j}^{2} P_{k+2 q^{\prime}, h}^{*}\right)
\end{aligned}
$$

where the last pumping term is only acting on the state with $k=k_{0}$ and $i=i_{0}$. The energies $E_{L P}$ are modified due to the exciton-exciton interaction and blueshifted $E_{L P}^{(i)}(k) \rightarrow$ $E_{L P}^{(i)}(k)+2 V_{k, k+q, k}^{i j h}\left|P_{k+q, j}\right|^{2}$. The interaction potential can be written as

$$
E_{i}^{h i j}(k)=\frac{1}{2}\left(V_{k, k, q}^{h i j}-V_{k, k,-q}^{h i j}\right) .
$$

Numerical simulations of the energy-momentum space distribution of the polariton occupation are shown in Fig. 2 (middle) for different excitation powers. We have chosen parameter values typical for $\mathrm{ZnO}$ or which we have derived from the experiment (as discussed above). The numerical results reproduce the experimental observations very well and thus confirm the model of parametric mixing of polariton 
states. Especially, the occupation of more and more low-energy states with increasing excitation power and the two main relaxation paths with its discrete energy-momentum states are reproduced. An obvious difference between the experimental observations and the modeling is the high occupation of polariton modes with energies above the excitation energy. This is caused by the limited number of dispersions taken into account in the simulations in order to maintain reasonable computation times. Furthermore, the polariton dispersions used are fitted using a coupled oscillator model, which enables one to assign angle- and mode-dependent photonic and excitonic fractions to the dispersions. We assume that all WGM modes have the same quality factor $Q=8000$ and an exciton-photon coupling of $80 \mathrm{meV}$. This very simplified approach results in high excitonic fractions of polariton states with high energies and thus increases the parametric scattering into these states. However, both reasons for the accumulation of polaritons in these high-energy modes do not affect the relaxation into the lower polariton states since no direct scattering channels are allowed.

In conclusion, polariton condensation accompanied by parametric polariton mixing has been experimentally observed and theoretically simulated in a one-dimensional multimode whispering gallery mode polariton system. The initial mode is resonantly excited by the radiative decay of defect-bound excitons, energetically far away from the free and donor-bound exciton transitions. With increasing excitation power, the parametric mixing process causes cooling of the polariton system by an effective energetic net relaxation, accompanied by momentum gain for low-energy states. The observed scattering properties resemble well the requirements for the creation of branch entangled polaritons. Especially, the intersubbranch scattering solely in the lower polariton branches and the internal resonant excitation of the initial mode at $k_{\|}=0$ provides a polariton system that is well isolated from the excitonic reservoir, as discussed in Ref. [8]. Thus we propose that one-dimensional $\mathrm{ZnO}$ microwires provide an excellent system for polariton lasing and entanglement as a source of entangled photons for future integrated quantum computing and communication applications.

This work has been supported by Leipzig School of Natural Sciences BuildMoNa (GS 185/1), the European Social Fund (ESF) within Nachwuchsforschergruppe "Multiscale functional structures," and DFG-FOR1616 (SCHM2710/2-1,P1) "Dynamics and Interactions of Semiconductor Nanowires for Optoelectronics." We gratefully acknowledge target preparation by G. Ramm.
[1] A. Amo, J. Lefrère, S. Pigeon, C. Adrados, C. Ciuti, I. Carusotto, R. Houdré, E. Giacobino, and A. Bramati, Superfluidity of polaritons in semiconductor microcavities, Nat. Phys. 5, 805 (2009).

[2] K. G. Lagoudakis, M. Wouters, M. Richard, A. Baas, I. Carusotto, R. André, Le Si Dang, and B. Deveaud-Plédran, Quantized vortices in an exciton-polariton condensate, Nat. Phys. 4, 706 (2008).

[3] K. G. Lagoudakis, T. Ostatnický, A. V. Kavokin, Y. G. Rubo, R. André, and B. Deveaud-Plédran, Observation of half-quantum vortices in an exciton-polariton condensate, Science 326, 974 (2009).

[4] S. Utsunomiya, L. Tian, G. Roumpos, C. W. Lai, N. Kumada, T. Fujisawa, M. Kuwata-Gonokami, A. Löffler, S. Höfling, A. Forchel, and Y. Yamamoto, Observation of bogoliubov excitations in exciton-polariton condensates, Nat. Phys. 4, 700 (2008).

[5] G. Dasbach, M. Schwab, M. Bayer, D. N. Krizhanovskii, and A. Forchel, Tailoring the polariton dispersion by optical confinement: Access to a manifold of elastic polariton pair scattering channels, Phys. Rev. B 66, 201201(R) (2002).

[6] V. Ardizzone, M. Abbarchi, T. Lecomte, A. Lemaitre, I. Sagnes, P. Senellart, J. Bloch, P. Roussignol, and J. Tignon, Optical parametric oscillaton in 1D semiconductor microcavities, Phys. Status Solidi B 249, 896 (2012).

[7] M. Abbarchi, A. Amo, V. G. Sala, D. D. Solnyshkov, H. Flayac, L. Ferrier, I. Sagnes, E. Galopin, A. Lemaître, G. Malpuech, and J. Bloch, Macroscopic quantum self-trapping and Josephson oscillations of exciton polaritons, Nat. Phys. 9, 275 (2013).

[8] C. Ciuti, Branch-entangled polariton pairs in planar microcavities and photonic wires, Phys. Rev. B 69, 245304 (2004).
[9] L. Einkemmer, Z. Vörös, G. Weihs, and S. Portolan, Entanglement generation in microcavity polariton devices, arXiv:1305.1469

[10] T. C. H. Liew and V. Savona, Multimode entanglement in coupled cavity arrays, New J. Phys. 15, 025015 (2013).

[11] E. Wertz, L. Ferrier, D. D. Solnyshkov, R. Johne, D. Sanvitto, A. Lemaître, I. Sagnes, R. Grousson, A. V. Kavokin, P. Senellart, G. Malpuech, and J. Bloch, Spontaneous formation and optical manipulation of extended polariton condensates, Nat. Phys. 6 , 860 (2010).

[12] C. P. Dietrich, M. Lange, C. Sturm, R. Schmidt-Grund, and M. Grundmann, One- and two-dimensional cavity modes in $\mathrm{ZnO}$ microwires, New J. Phys. 13, 103021 (2011).

[13] T. Nobis, E. M. Kaidashev, A. Rahm, M. Lorenz, and M. Grundmann, Whispering gallery modes in nanosized dielectric resonators with hexagonal cross section, Phys. Rev. Lett. 93, 103903 (2004).

[14] C. P. Dietrich, M. Brandt, M. Lange, J. Kupper, T. Böntgen, H. von Wenckstern, and M. Grundmann, Defect properties of $\mathrm{ZnO}$ and ZnO:P microwires, J. Appl. Phys. 109, 013712 (2011).

[15] B. K. Meyer, H. Alves, D. M. Hofmann, W. Kriegseis, D. Forster, F. Bertram, J. Christen, A. Hoffmann, M. Strassburg, M. Dworzak, U. Haboeck, and A. V. Rodina, Bound exciton and donor-acceptor pair recombinations in $\mathrm{ZnO}$, Phys. Status Solidi B 241, 231 (2004).

[16] See Supplemental Material at http://link.aps.org/supplemental/ 10.1103/PhysRevB.91.041202 for details about sample fabrication, setup, and further properties of the condensate.

[17] A. Trichet, E. Durupt, F. Médard, S. Datta, A. Minguzzi, and M. Richard, Long-range correlations in a $97 \%$ excitonic one- 
dimensional polariton condensate, Phys. Rev. B 88, 121407(R) (2013).

[18] D. M. Coles and D. G. Lidzey, A ladder of polariton branches formed by coupling an organic semiconductor exciton to a series of closely spaced cavity-photon modes, Appl. Phys. Lett. 104, 191108 (2014).

[19] M. Wouters, T. C. H. Liew, and V. Savona, Energy relaxation in one-dimensional polariton condensates, Phys. Rev. B 82, 245315 (2010).

[20] S. Acharya, S. Chouthe, H. Graener, T. Böntgen, C. Sturm, R. Schmidt-Grund, M. Grundmann, and G. Seifert, Ultrafast dynamics of the dielectric functions of $\mathrm{ZnO}$ and $\mathrm{BaTiO} 3$ thin films after intense femtosecond laser excitation, J. Appl. Phys. 115, 053508 (2014).

[21] A. Müller and M. Grundmann, Tunneling dynamics of excitons in random semiconductor alloys, Phys. Rev. B 87, 035134 (2013).
[22] M. Maragkou, A. J. D. Grundy, E. Wertz, A. Lemaître, I. Sagnes, P. Senellart, J. Bloch, and P. G. Lagoudakis, Spontaneous nonground state polariton condensation in pillar microcavities, Phys. Rev. B 81, 081307(R) (2010).

[23] D. G. Thomas, The exciton spectrum of zinc oxide, J. Phys. Chem. Solids 15, 86 (1960)

[24] C. Czekalla, T. Nobis, A. Rahm, B. Q. Cao, J. Zúñiga-Pérez, C. Sturm, R. Schmidt-Grund, M. Lorenz, and M. Grundmann, Whispering gallery modes in zinc oxide micro- and nanowires, Phys. Status Solidi B 247, 1282 (2010).

[25] C. Ciuti, P. Schwendimann, B. Deveaud, and A. Quattropani, Theory of the angle-resonant polariton amplifier, Phys. Rev. B 62, R4825 (2000).

[26] C. Ciuti, P. Schwendimann, and A. Quattropani, Theory of polariton parametric interactions in semiconductor microcavities, Semicond. Sci. Technol. 18, S279 (2003). 